\title{
On Resource Allocation for Machine-to-Machine (M2M) Communications in Cellular Networks
}

\author{
Harpreet S. Dhillon, Howard C. Huang, Harish Viswanathan and Reinaldo A. Valenzuela
}

\begin{abstract}
Cellular networks are an attractive option for handling the growing number of sensing and monitoring devices due to their ubiquitous presence. While this growing popularity of cellular network based machine-to-machine (M2M) communications is opening new avenues for the mobile network operators, it is also bringing forth new system design challenges mainly because of the significant difference in the nature of M2M traffic and the current commercial traffic for which the cellular networks are designed and optimized. In this paper, we consider the M2M operational regime characterized by large number of small transactions and study the problem of power optimal uplink resource allocation both for Time Division Multiple Access (TDMA) and Frequency Division Multiple Access (FDMA). We derive tractable results for the maximum load a base station can handle and the optimal transmit power for both access strategies and show that FDMA supports an order of magnitude higher load than TDMA under the peak power constraint. We also show that the value of optimizing uplink resource allocation in the M2M parameter space of interest is typically insignificant and simpler access strategies, such as channel gain based allocation or even equal resource allocation, lead to near optimal performance. We also derive accurate closed form approximations for optimum power levels indicative of the actual performance in this regime.
\end{abstract}

\section{INTRODUCTION}

Machine-to-machine (M2M) communications, involving wide area communication of sensor data to an Internet based application, is emerging as an important paradigm in mobile cellular networks [1]-[3]. With diminishing voice revenues, mobile network operators are increasingly relying on data services for revenue growth with M2M being a major area of focus within data services [4]. Projections for growth of M2M communication devices range from 24 Billion [5] to 50 Billion [6] in the next decade with over 2 Billion M2M devices expected to directly attach to the cellular network by this time. M2M communications span multiple vertical industries such as transportation, healthcare, utilities, retail, industrial monitoring, banking and home automation, and include a variety of applications within each vertical.

M2M traffic is typically distinct from consumer traffic and is characterized by large number of short payload transactions [7], [8]. For example, a fleet management application can involve transmission of location every 20 seconds by each vehicle to the central application server with each transaction involving less than 500 bytes [9]. Similarly, reporting of health data such as blood pressure or heart rate by medical

H. S. Dhillon is with WNCG, the University of Texas at Austin, USA. Email: dhillon@utexas.edu. H. C. Huang, H. Viswanathan and R. A. Valenzuela are with Bell Labs, Alcatel-Lucent, NJ. Email: \{howard.huang, harish.viswanathan, reinaldo.valenzuela\}@alcatel-lucent.com. devices involves payloads less than 200 bytes [10]. On the other hand, mobile cellular networks, including the fourth generation Long Term Evolution (LTE), have been designed for handling consumer communication applications such as voice, video streaming, video conferencing, web browsing, and file transfers where the communication sessions are fairly long lived. Thus a typical session involves establishment of radio bearers by first using a common random access channel (RACH) to send relevant control information such as identity of the device followed by actual transmission of higher layer signaling messages and then application data over scheduled dedicated resources. This clearly results in disproportionately large amount of signaling traffic relative to data traffic if the current cellular communication technology is used as it is for M2M applications.

The above difference in the operational regimes imposes new requirements on cellular networks that demand rethinking some of the design principles [11], [12]. Motivated by this observation, several modifications in the current communication protocols to reduce signaling overhead [13]-[15] and power consumption [16] have been proposed in the literature. Furthermore, the idea of cooperative design where several devices are clustered together with a possibility of a controller acting as a common link between a cellular base station and the devices is investigated, e.g., in [17], [18]. For the current paper, perhaps the most relevant prior work is [19], which studies channel and delay aware uplink scheduling for M2M devices in LTE networks. However, the proposed algorithms are heuristic and provide a very little fundamental understanding of uplink resource allocation in M2M regime especially from a power optimization perspective. Since the devices are typically battery operated and it may not always be easy to replace or recharge these batteries, optimizing transmit power is an important consideration and will be the main focus of this paper.

Minimum energy and/or power scheduling is known to be a convex optimization problem and various efficient algorithms have been proposed in the literature [20]-[22]. We show that, in the cellular setting, it is possible to approximate the optimal schedule through simpler one-shot solutions, which leads to the closed form expressions for the optimization parameters in certain special cases. Furthermore, we prove that in the parameter space of interest, somewhat surprisingly, simple equal time allocation performs within a factor of 2 of the optimal solution for minimizing the transmission power in TDMA. A similar result is numerically shown to hold for the average transmit power required in the FDMA case, thereby suggesting that a simple resource allocation 


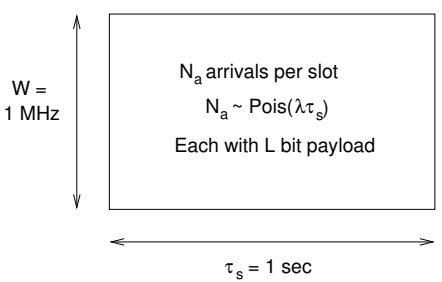

Fig. 1. Illustration of the time-frequency resource "slice" over which multiple users are scheduled.

algorithm is sufficient to achieve near-optimum transmission in terms of power minimization. We also derive accurate closed form approximations for the optimum power levels that are indicative of the actual performance in this regime.

\section{SYSTEM MODEL}

In this paper, we consider a single cell consisting of a base station lying at the origin and devices uniformly distributed around it in an annular region with inner and outer radii $r_{i}$ and $r_{0}$, respectively. The non-zero inner radius is assumed to avoid singularity in the path loss model, which is discussed later in this section. We ignore out-of-cell interference, which is also one of the assumptions of the 3GPP model [11]. It should be noted that the out-of-cell interference effectively changes the operating signal-to-interference-plus-noise ratio (SINR), which can be incorporated in the current analysis to study the multi-cell case. To characterize the uplink load seen by the base station, we model the arrival process of packets as a Poisson point process with mean $\lambda$ packets per second. For concreteness, we assume a time slotted system with the slot duration denoted by $\tau_{s}$. The analysis will be performed on a typical time-frequency resource "slice" with slot duration $\tau_{s}$ and bandwidth $W$ as shown in Fig. 1. For numerical arguments, we will consider $\tau_{s}=1 \mathrm{sec}$ and $W=1$ $\mathrm{MHz}$. We denote the number of packet transmission requests in each slice by $N_{a} \sim \operatorname{Pois}\left(\lambda \tau_{s}\right)$. Each packet is assumed to have a payload of $L$ bits.

We further assume that the base station has complete channel state information (CSI) through the preceding random access channel $(\mathrm{RACH})$ stage which is not discussed in this paper. To incorporate an explicit notion of packet deadlines, we assume that all packet transmissions occur in the current slot and none of them are left to be scheduled in a future slot. This leads to the deadline of $\tau_{s}$ for each packet. Our goal is to study non-overlapping power optimal schedules for $N_{a}$ packets in this setup and gain insights into the maximum load a base station can handle using TDMA or FDMA.

For the link budget, instead of treating the parameters individually, we model their composite effect by defining reference Signal-to-Noise-Ratio (SNR) $\mu$ as the average SNR at the cell edge or at half the minimum inter-site distance (assuming hexagonal geometry). We will take $\mu=-3 \mathrm{~dB}$ unless explicitly mentioned. While defining $\mu$, we assume the signal is transmitted with the maximum transmit power $P_{\max }$ (assumed $1 \mathrm{~W}$, i.e., $30 \mathrm{dBm}$ ) over bandwidth $W$, unit antenna gains and ignore fading and shadowing effects. Please note that reference SNR is a function of signal bandwidth because of the scaling of noise power with bandwidth. Under these assumptions, the uplink received power of a device located at distance $r$ from the base station is:

$$
P_{r}=P_{t} P_{0} \mathcal{X} h\left(\frac{r}{r_{0}}\right)^{-\gamma} G,
$$

where $P_{t}$ is the transmit power, $P_{0}$ is the received power at the reference distance (assuming unit transmit power), $\mathcal{X}$ is the large-scale shadowing random variable, $h$ is the small-scale fading random variable, $r_{0}$ is the reference distance which is defined as half of the inter-site distance, also the outer radius of the annular region defined in the system setup, $\gamma$ is the pathloss exponent and $G$ is the antenna gain. Defining channel gain $g$ as $g=\mathcal{X} h\left(\frac{r}{r_{0}}\right)^{-\gamma} G$ and keeping in mind that effectively both $P_{0}$ and $\mu$ are defined by assuming unit transmit power, we can write the received SNR $\mu_{r}$ as:

$$
\mu_{r}=P_{t} \mu g
$$

In case the information symbols are transmitted over a bandwidth $W_{\mathcal{N}}<W$, the reference SNR $\mu_{\mathcal{N}}$ in this case can be written in terms of $\mu$ as:

$$
\mu_{\mathcal{N}}=\mu \frac{W}{W_{\mathcal{N}}}
$$

which is greater than $\mu$ because of the decrease in the effective noise power. For this modified system, the received SNR can be evaluated from (2) by replacing $\mu$ with $\mu_{\mathcal{N}}$. This insight will be helpful in analyzing FDMA that involves partitioning over the frequency resources.

Depending upon the choice of resource partitioning strategy, information symbols will be transmitted over bandwidth $W_{\mathcal{N}} \leq W$ for time $\tau \leq \tau_{s}$. Assuming capacity achieving codes, $\tau$ and $W_{\mathcal{N}}$ are related to the received SNR $\mu_{r}$ by Shannon's capacity equation as follows:

$$
\frac{L}{\tau W_{\mathcal{N}}}=\log _{2}\left(1+\mu_{r}\right) .
$$

It should be noted that the effect of finite block length can be easily incorporated in the above expression by means of an SNR gap. Interested readers can refer to [23] for more details. Using (2) and (4), we can find the minimum transmission time required to transmit $L$ bits over bandwidth $W_{\mathcal{N}}$ under the maximum transmit power $P_{\max }$ constraint as:

$$
\tau \geq \frac{L}{W_{\mathcal{N}} \log _{2}\left(1+P_{\max } \mu_{\mathcal{N}} g\right)}=\tau_{\min } .
$$

Similarly, the minimum transmission bandwidth required for the transmission of $L$ bits over time $\tau$ is given by the solution of the following equation:

$$
\frac{L}{\tau W_{\min }}=\log _{2}\left(1+P_{\max } \mu\left(\frac{W}{W_{\min }}\right) g\right) .
$$

As will be explained later, (5) and (6) would be useful in formulating the optimization problems for TDMA and FDMA cases, respectively. 


\section{MAXimum LoAd For Power Optimal Scheduling}

As is clear from (5) and (6) derived in Section II, there is a minimum transmission time $\tau_{\min }$ or minimum bandwidth $W_{\min }$ required for the successful transmission of a packet, which appears due to the constraint on maximum power at which a device can transmit. Because of these constraints on the minimum resources required, there is clearly a fundamental limit on the number of packets that can be scheduled in a given time-frequency resource slice. Beyond this limit, the base station would not be able to schedule all the packets without failures. This limit defines the maximum load a base station can handle and is dependent upon the resource partitioning strategy being employed. Since the devices farther out near the cell edge or in deep fade require more resources than the others, we assume that the base station drops a small fraction of these packets to increase the maximum load it can handle and to reduce the overall average transmit power [24].

For concreteness, we assume that the base station serves $K=(1-\delta) N_{a}$ packets where $\delta$ is the maximum outage allowed by the system. It should be noted that the dropped packets are the ones having smallest channel gains. For resource partitioning, we consider two approaches: i) TDMA - splitting time slot $\tau_{s}$ into $K$ parts, and ii) FDMA - splitting bandwidth $W$ into $K$ parts, where the goal in both approaches is to minimize total transmit power. As shown later in this section, the optimization problem to find the power optimal schedule is convex and hence can be solved efficiently using known algorithms, such as the MoveRight algorithm [21]. Besides, we show that numerical optimization is not necessary since a near-optimal tractable solution can be found where the resources allocated to each device solely depend upon its channel gain and are independent of the channel gains of other devices. More interestingly, we analytically show that equal resource allocation compares quite favorably with the optimal schedule and might as well be preferred by the system designers because of its apparent simplicity.

In this discussion, we will assume non-overlapping transmissions, i.e., the resources are partitioned such that a part of the resource is allocated only to a single device. We first discuss the optimal schedules for TDMA in detail and then show that the results for FDMA directly follow.

\section{A. Optimal Schedule for TDMA}

As stated above, our first goal is to find an optimal partition of $\tau_{s}=\left\{\tau_{1}, \tau_{2}, \ldots, \tau_{K}\right\}$ so as to minimize total transmit power. Therefore, each device transmits its $L$ bit information over bandwidth $W$ in time $\tau_{i}$. The optimization problem can be formulated as follows:

$$
\begin{array}{ll}
\min _{\left\{\tau_{i}\right\}} & \sum_{i=1}^{K} \frac{2^{\frac{L}{W \tau_{i}}}-1}{\mu g_{i}} \\
\text { s.t. } & \sum_{i=1}^{K} \tau_{i} \leq \tau_{s} \\
& \tau_{i} \geq \frac{L}{W \log _{2}\left(1+P_{\max } \mu g_{i}\right)}\left(=\tau_{\min _{i}}\right) \\
& 1 \leq i \leq K,
\end{array}
$$

where the cost function follows from Shannon's capacity expression and the $\tau_{\min }$ constraint from (5). Clearly the optimization problem is convex and can be efficiently solved using known algorithms.

Remark 1 (Feasibility and Maximum Load). The above optimization problem is feasible if the constraint $\sum_{i=1}^{K} \tau_{\min _{i}} \leq \tau_{s}$ is satisfied. Using this constraint, an approximation on the maximum load can be derived as follows.

$$
\frac{\sum_{i=1}^{K} \tau_{\min _{i}}}{K} \leq \frac{\tau_{s}}{K} \Rightarrow K \leq \frac{\tau_{s}}{\sum_{i=1}^{K} \tau_{\min _{i}} / K} \stackrel{(a)}{\approx} \frac{\tau_{s}}{\mathbb{E}\left[\tau_{\min }\right]},
$$

where (a) follows from two approximations: i) Law of Large Numbers (LLN) holds and the average converges to the mean of the random variables, ii) the mean is $\mathbb{E}\left[\tau_{\min }\right]$, which is not exact because $\delta N_{a}$ packets with smallest channel gains have been removed and hence order statistics should be used to compute the mean of the remaining random variables. Nevertheless, the first approximation is tight when $K$ is large and second is tight when $\delta \rightarrow 0$, which is essentially the regime of interest for a realistic system design. The maximum load $\lambda_{\max }$ is now given by:

$$
\lambda_{\max }=\max _{\lambda}\left\{\mathbb{P}\left((1-\delta) N_{a} \geq \frac{\tau_{s}}{\mathbb{E}\left[\tau_{\min }\right]}\right) \rightarrow 0\right\} .
$$

1) Near Optimal Closed-Form Solution: The form of the optimization problem is such that the exact closed form solutions are not possible. We now show that it is possible to derive approximate near-optimal closed form results, where the transmission time is solely a function of the channel gain of the device of interest independent of the channel gains of other devices, which also leads to a simpler resource allocation strategy that circumvents the need for numerical optimization. The total transmit power can be expressed as:

$$
\begin{aligned}
P= & \sum_{i=1}^{K} \frac{2^{\frac{L}{W \tau_{i}}}-1}{\mu g_{i}}=\frac{2^{\frac{L}{W \tau_{1}}}-1}{\mu g_{1}}+\frac{2^{\frac{L}{W \tau_{2}}}-1}{\mu g_{2}} \\
& \ldots \frac{2^{W\left(\tau_{s}-\sum_{j=1}^{K-1} \tau_{j}\right)}-1}{\mu g_{K}} .
\end{aligned}
$$

Minimizing the transmit power $P$ w.r.t. $\tau_{1}$ we get

$$
\frac{\delta P}{\delta \tau_{1}}=0 \Rightarrow \frac{2^{\frac{L}{W \tau_{1}}}}{g_{1} \tau_{1}^{2}}=\frac{2^{\frac{L}{W \tau_{K}}}}{g_{K} \tau_{K}^{2}} \Rightarrow \tau_{i}^{2} 2^{\frac{-L}{W \tau_{i}}} \propto g_{i}^{-1}
$$

Remark 2. For small $\lambda, \tau$ is of the order of seconds, and for our choice of $L$ and $W, L / W=10^{-3}$, which implies $2^{\frac{-L}{W \tau}} \rightarrow 1$. Therefore, $\tau \propto g^{-1 / 2}$. On the other hand, for high $\lambda($ say $\lambda=1000)$, and $L / W=10^{-3}$, we have $2^{\frac{L}{W \tau}} \approx \frac{2 L}{W \tau}$. Therefore, $\tau \propto g^{-1 / 3}$ in this regime.

From the above remark, we note that the transmission time can be expressed solely as a function of channel gain as

$$
\tau_{i}=\frac{f\left(g_{i}\right)}{\sum_{j} f\left(g_{j}\right)} \tau_{s},
$$

which reduces further to

$$
\tau_{i} \approx \frac{f\left(g_{i}\right)}{\lambda E[f(g)]},
$$


for reasonably high values of $\lambda$. Ignoring fading and shadowing and assuming $f\left(g_{i}\right)=g_{i}^{-1 / n}$ that encompasses both the special cases of $n=2$ and $n=3$, (12) can be expressed in the closed form as follows:

$$
\tau_{i}=\frac{2+\gamma / n}{2 \lambda} r^{\gamma / n} \frac{r_{0}^{2}-r_{i}^{2}}{r_{0}^{2+\gamma / n}-r_{i}^{2+\gamma / n}} .
$$

In Fig. 2, we plot the closed form result (13) along with a scatter plot of the optimal transmission times in two regimes: i) low load $(\lambda=10)$ and ii) high load $(\lambda=1000)$. We note that the approximations for both regimes are quite accurate, which is more surprising for the low load case since the simplification of (11) to (12) holds only for high $\lambda$. Nevertheless, we will comment further on the accuracy of this simple approach in the numerical results section.
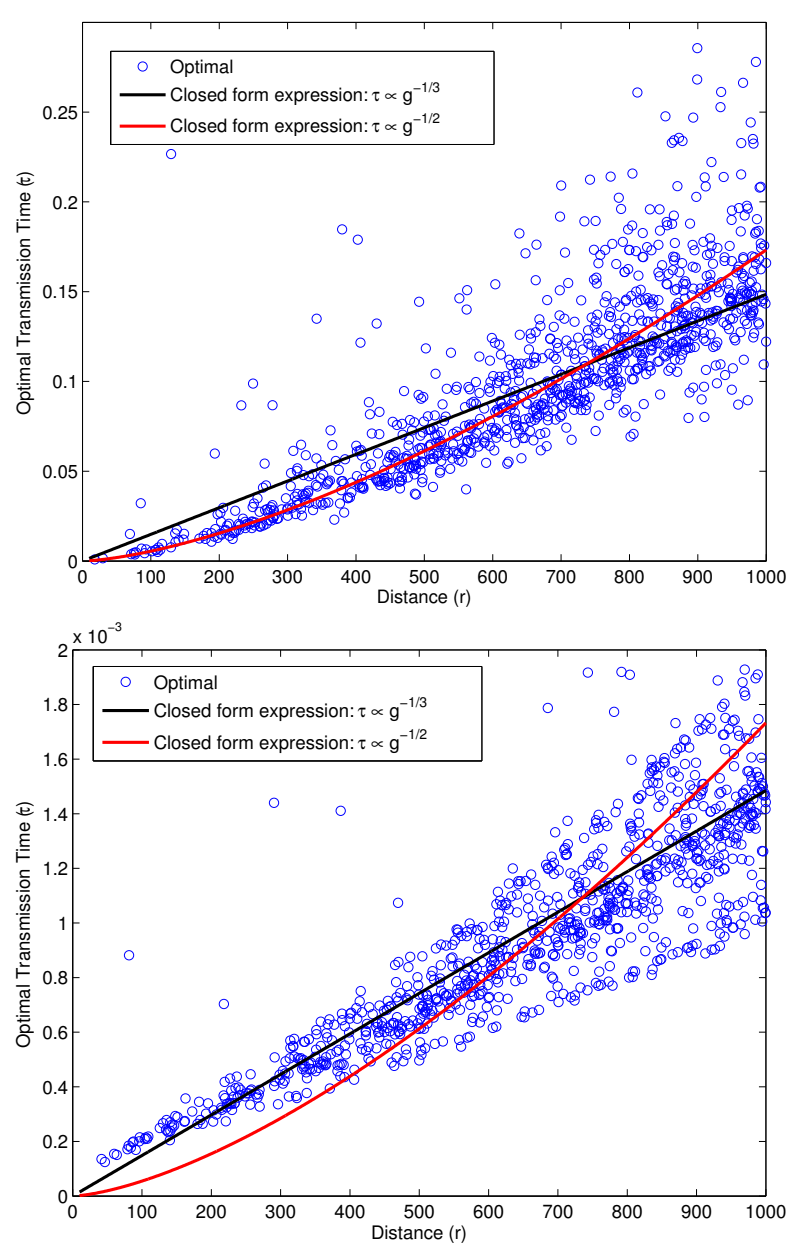

Fig. 2. Comparison of the closed form solution given by (13) with the power optimal solution (scatter plot). (left) low load $(\lambda=10)$. (right) high load $(\lambda=1000)$. The fading and shadowing are ignored.

2) Equal Time Allocation vs Power Optimal Schedule: We now consider an even simpler case in which each device is allocated the same transmission time and show that this simple solution compares favorably with the optimal solution.

Let $U_{g_{1}, g_{2} \ldots g_{K}}$ denote the total transmit power under uniform schedule (equal transmission time) and $P_{g_{1}, g_{2} \ldots g_{K}}$ denote the power under the optimal schedule. Further let $U_{g_{i}, \ldots g_{j}}$ and $P_{g_{i}, \ldots g_{j}}$ be the transmit powers of the subset of users under uniform and optimal schedules, respectively. Without loss of generality, we assume that the channel gains $g_{i}$ are indexed in the increasing orders of magnitude. Let the total transmission time of $K$ devices be equal to the slot length $\tau_{s}$, with $\tau_{j}^{*}$ representing the optimal transmission time of $j^{\text {th }}$ packet.

Theorem 1. The ratio of total transmit powers under uniform and optimal schedules can be bounded as:

$$
\frac{U_{g_{1}, g_{2} \ldots g_{K}}}{P_{g_{1}, g_{2} \ldots g_{K}}} \leq \frac{2^{\frac{K L}{W \tau_{s}}}-1}{2^{\frac{\sum_{j=1}^{K} \sqrt{\frac{g_{1}}{g_{j}}}}{W \tau_{s}}}-1},
$$

where $g_{1}=\min \left\{g_{j}\right\}$.

Proof: The ratio of powers under two scheduling schemes can be expressed as:

$$
\begin{aligned}
\frac{U_{g_{1}, g_{2} \ldots g_{K}}}{P_{g_{1}, g_{2} \ldots g_{K}}} & =\frac{U_{g_{1}}+U_{g_{2}}+\ldots U_{g_{K}}}{P_{g_{1}}+P_{g_{2}}+\ldots P_{g_{K}}} \\
& \leq \max \left\{\frac{U_{g_{1}}}{P_{g_{1}}}, \frac{U_{g_{2}}}{P_{g_{2}}}, \ldots, \frac{U_{g_{K}}}{P_{g_{K}}}\right\}
\end{aligned}
$$

The ratio $U_{g_{k}} / P_{g_{k}}$ can be expressed as:

$$
\frac{U_{g_{k}}}{P_{g_{k}}}=\frac{2^{\frac{K L}{W \tau_{s}}}-1}{2^{\frac{L}{W \tau_{k}^{*}}}-1} .
$$

From the following optimality condition derived in (10):

$$
\frac{2^{L / W \tau_{k}^{*}}}{g_{k} \tau_{k}^{*^{2}}}=\frac{2^{L / W \tau_{1}^{*}}}{g_{1} \tau_{1}^{*^{2}}},
$$

we note that $\tau_{1}^{*} \geq \tau_{k}^{*} \forall k$ since $g_{1} \leq g_{k}$. Therefore, (16) can be expressed as:

$$
\frac{U_{g_{1}, g_{2} \ldots g_{K}}}{P_{g_{1}, g_{2} \ldots g_{K}}} \leq \frac{U_{g_{1}}}{P_{g_{1}}} .
$$

To bound $\frac{U_{g_{1}}}{P_{g_{1}}}$, we first derive the following inequality from the optimality condition:

$$
\tau_{k}^{*} \geq \tau_{1}^{*} \sqrt{\frac{g_{1}}{g_{k}}}, \forall k .
$$

Using (20), we now derive an upper bound on $\tau_{1}^{*}$ as follows:

$$
\tau_{s}=\sum_{j=1}^{K} \tau_{j}^{*} \geq \tau_{1}^{*} \sum_{j=1}^{K} \sqrt{\frac{g_{1}}{g_{j}}},
$$

which implies:

$$
\tau_{1}^{*} \leq \tau_{s} / \sum_{j=1}^{K} \sqrt{\frac{g_{1}}{g_{j}}} .
$$

Using (22), the ratio $U_{g_{1}} / P_{g_{1}}$ can be bounded as:

$$
\frac{U_{g_{1}}}{P_{g_{1}}}=\frac{2^{K L / W \tau_{s}}-1}{2^{L / W \tau_{1}^{*}}-1} \leq \frac{2^{\frac{K L}{W \tau_{s}}}-1}{2^{\frac{\sum_{j=1}^{K} \sqrt{\frac{g_{1}}{g_{j}}}}{W \tau_{s}}}-1},
$$

which completes the proof.

Example 1 (Bound in the parameter space of interest). For $K=1000, \tau_{s}=1 \mathrm{sec}, L=1000, W=1 \mathrm{MHz}$ and no fading, the bound given by (14) is $\approx 2$, i.e., it is guaranteed that the transmit power under uniform scheduling 
is no more than around $3 d B$ worse than the optimal power. In the numerical results section, we show that the simple equal resource allocation solution is even more accurate than predicted by this bound.

\section{B. FDMA System Design}

The FDMA system design proceeds exactly in the same way as discussed for the TDMA case above. We will therefore highlight only the main differences in problem formulation. The goal here is to partition the available bandwidth $W$ into $K$ parts $\left\{W_{1}, W_{2}, \ldots, W_{K}\right\}$ so as to minimize the total transmit power. The optimization problem can be formulated as follows:

$$
\begin{array}{ll}
\min _{\left\{W_{i}\right\}} & \sum_{i=1}^{K} \frac{2^{\frac{L}{W_{i} \tau_{s}}}-1}{\mu W g_{i}} \\
\text { s.t. } & \sum_{i=1}^{K} W_{i} \leq W \\
& W_{i} \geq W_{\text {min }_{i}} \\
& 1 \leq i \leq K,
\end{array}
$$

where the cost function again follows from the Shannon's capacity expression and the minimum bandwidth $W_{\min }$ is the solution of (6) for $\tau=\tau_{s}$. We note that the form of the optimization problem is exactly the same as that of the TDMA problem discussed above in detail and hence most of the insights about the exact and approximate solutions carry over.

Remark 3 (Feasibility and Maximum Load). As discussed for the TDMA counterpart in Remark 1, the optimization problem is feasible if the constraint $\sum_{i=1}^{K} W_{\min _{i}} \leq W$ is satisfied. Using this constraint, an approximate bound on $K$ can be derived as:

$$
K \leq \frac{W}{\mathbb{E}\left[W_{\min }\right]},
$$

using which the maximum load $\lambda_{\max }$ can be expressed as:

$$
\lambda_{\max }=\max _{\lambda}\left\{\mathbb{P}\left((1-\delta) N_{a} \geq \frac{W}{\mathbb{E}\left[W_{\min }\right]}\right) \rightarrow 0\right\} .
$$

This completes the analysis of the power optimal schedules and we now compare the maximum load that can be handled by a base station using TDMA and FDMA in the following example.

Example 2 (Maximum Load: FDMA vs TDMA). For $L=$ $1000, W=1 \mathrm{MHz}, \tau_{s}=1 \mathrm{sec}, \mu=-3 \mathrm{~dB}, \delta=0$ and no fading, the maximum loads a base station can handle using TDMA (8) and FDMA $(25)$ are $\approx 1200$ and $\approx 14700$, respectively. This clearly shows that it is optimal to partition over frequency. Although TDMA and FDMA are exactly the same from information theoretic sense, the difference in the optimal solution arrises as a result of the peak power constraint that affects the two schemes differently as is apparent from the expressions of $\tau_{\min }$ and $W_{\min }$.

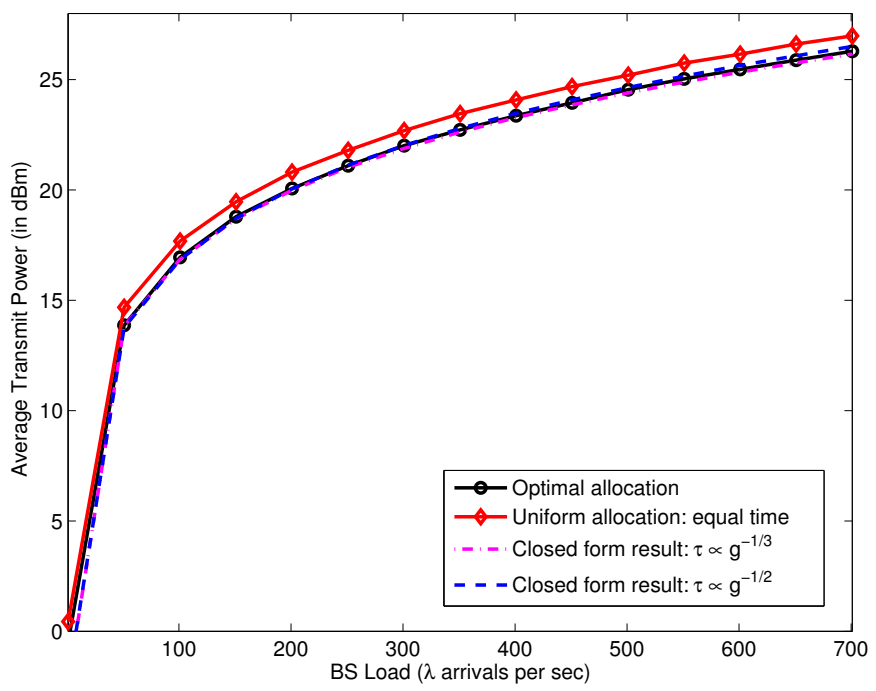

Fig. 3. Comparison of the TDMA optimal power solution with various special cases $(L=1000, W=1 \mathrm{MHz}, \mu=-3 \mathrm{~dB}, \gamma=3$, no fading).

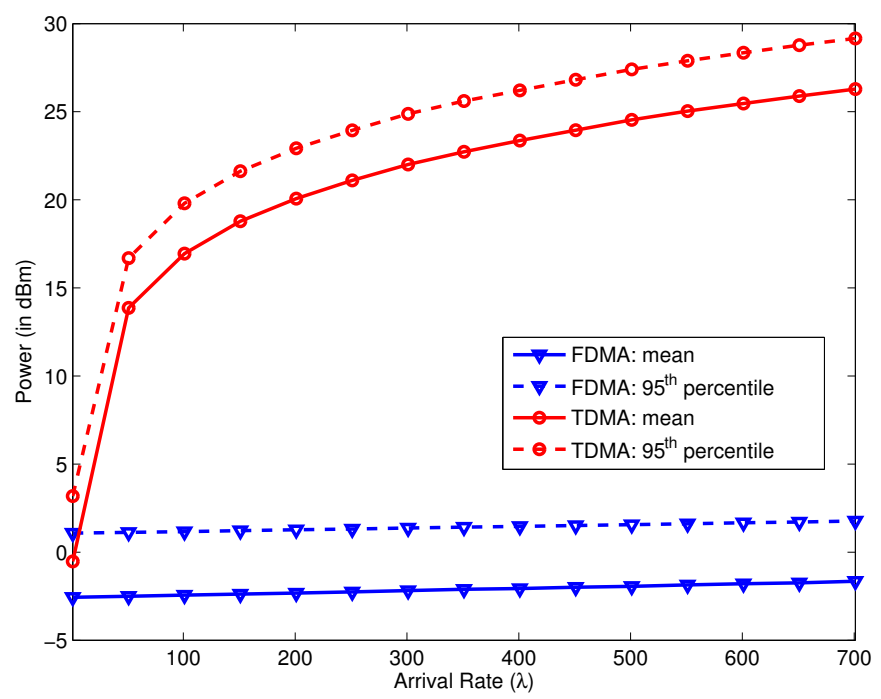

Fig. 4. Comparison of power optimal solutions for TDMA and FDMA.

\section{Numerical Results}

In this section, we focus on the accuracy of various approximate analytical results and the performance of sub-optimal schemes proposed in this paper. For numerical computations, we consider the same values for the system parameters as that of Example 2.

We first compare the average optimal power required in TDMA case with three special cases: i) equal time allocation, ii) closed form expression (13) for $n=2$ and, iii) closed form expression (13) for $n=3$. As seen earlier in Theorem 1, the equal time allocation strategy achieves near optimal performance. This circumvents the need for optimization in this regime and hence simplifies the system design. We also note that both theoretical expressions closely approximate the optimal solution found numerically and hence can be used as proxies for the optimal solution in this regime.

In Fig. 4, we compare the transmit powers associated with 


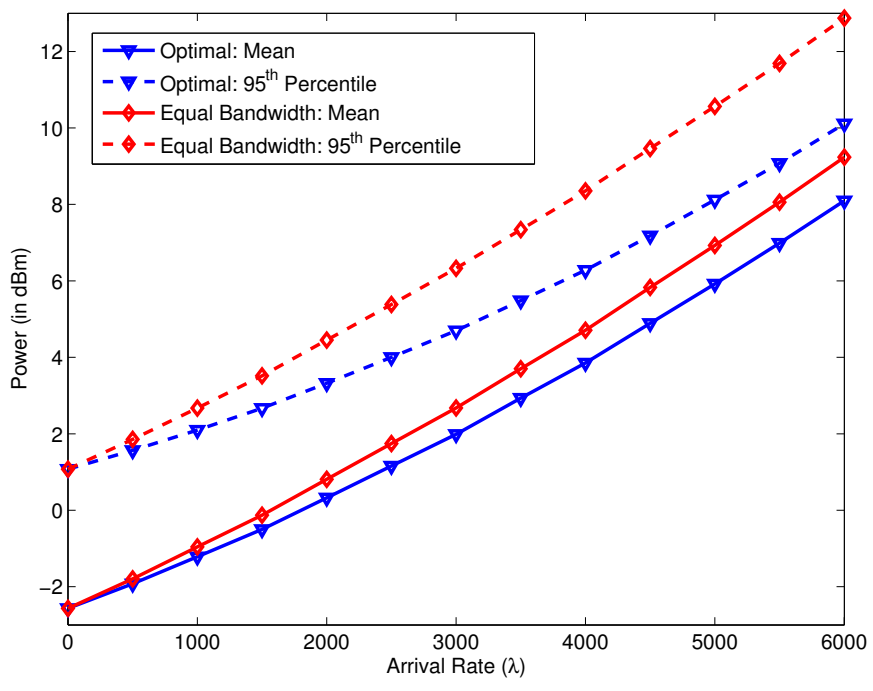

Fig. 5. Comparison of the optimal power and equal bandwidth allocation schedules for FDMA.

the optimal TDMA and FDMA schedules for low to moderate arrival rates. In addition to the average powers, we also compare the $95^{\text {th }}$ percentile of the transmit power to highlight the likely performance of the edge users. Both results clearly show that it is optimal to use FDMA in this regime. The huge difference in the transmit powers of the two cases also corroborate the findings of Example 2, where FDMA was shown to support a much higher arrival rate than TDMA.

In Fig. 5 we compare the optimal bandwidth allocation strategy with equal resource allocation for FDMA over a large range of arrival rates. As was the case in TDMA, the equal resource allocation strategy is near-optimal in terms of transmit power minimization. However, when we compare the $95^{\text {th }}$ percentile of the transmit power, we note that there is non-negligible difference in the two strategies especially at very high arrival rates, e.g., at $\lambda=6000$ the equal allocation strategy requires around $3 \mathrm{~dB}$ more transmit power. Equivalently, the optimal schedule allows $\approx 1500-2000$ more packets per second than the equal resource allocation case. Please note that implementation complexity of the RF front end for both the cases is the same since the transmission bandwidth can not be fixed a priori in any of these cases.

\section{CONCLUSION}

In this paper, we have studied the power optimal uplink resource allocation problem both for TDMA and FDMA in the parameter range of interest for cellular network based M2M communications. We have analytically shown that i) FDMA supports an order of magnitude higher load than TDMA, and ii) the value of optimal resource allocation in the M2M regime is not significant and simple strategies such as equal resource allocation achieve reasonably good performance. This work has numerous extensions, e.g., we need to incorporate the fact that there will be limitations to the FDMA-only solution when the slot duration is very high because the transmission bandwidth becomes too small to be practical. We also did not consider RACH design, which is expected to be significantly different from the current LTE system due to the large number of simultaneous transmissions expected in M2M applications.

\section{REFERENCES}

[1] The FocalPoint Group, "M2M white paper: the growth of device connectivity," white paper, 2003, available online: goo.gl/S0j5O.

[2] Vodafone, "Global machine to machine communication," white paper, 2010, available online: goo.gl/4V8az.

[3] Ericsson, "Device connectivity unlocks value," white paper, Jan. 2011, available online: goo.gl/alou6.

[4] S.-Y. Lien, K.-C. Chen, and Y. Lin, "Toward ubiquitous massive accesses in 3GPP machine-to-machine communications," IEEE Communications Magazine, vol. 49, no. 4, pp. 66 - 74, Apr. 2011

[5] GSMA, "Experience a world where everything intelligently connects: The Connected Life," white paper, Feb. 2012, available online: goo.gl/gaqeD.

[6] Ericsson, "More than 50 billion connected devices," white paper, Feb. 2011, available online: goo.gl/vjYOO.

[7] H. Viswanathan, K. Mun, and F. Scholler, "Modeling and analysis of cellular wireless machine-to-machine communication traffic," submitted to IEEE Journal on Sel. Areas in Communications, Apr. 2012.

[8] M. Z. Shafiq, L. Ji, A. X. Liu, J. Pang, and J. Wang, "A first look at cellular machine-to-machine traffic - large scale measurement and characterization," London, Jun. 2012.

[9] Y. Jou, R. Attar, S. Ray, J. Ma, and X. Zhang, "M2M over CDMA2000 $1 \mathrm{x}$ case studies," in Proc., IEEE Wireless Communications and Networking Conf. (WCNC), Cancun, Mexico, Mar. 2011, pp. 1546 - 1551.

[10] Health Informatics - PoC Medical Device Communication - Part 00101: Guide-Guidelines for the Use of RF Wireless Technology, IEEE Std 11073-00101-2008, Dec. 2008.

[11] Study on RAN improvements for Machine-Type Communications, 3GPP TR 37.868, 2010

[12] K. Zheng, F. Hu, W. Wang, W. Xiang, and M. Dohler, "Radio resource allocation in LTE-advanced cellular networks with M2M communications," IEEE Communications Magazine, vol. 50, no. 7, pp. 184-192, Jul. 2012.

[13] M. Martsola, T. Kiravuo, and J. K. O. Lindqvist, "Machine to machine communication in cellular networks," in Proc., IEE Mobility Conference, Guangzhou, China, Nov. 2005.

[14] Y. Chen and Y. Yang, "Cellular based machine to machine communication with un-peer2peer protocol stack," in Proc., IEEE Veh. Technology Conf. (VTC), Anchorage, Alaska, Sep. 2009.

[15] Y. Chen and W. Wang, "Machine-to-machine communication in LTEA," in Proc., IEEE Veh. Technology Conf. (VTC), Ottawa, Canada, Sep. 2010.

[16] H. Chao, Y. Chen, and J. Wu, "Power saving for machine to machine communications in cellular networks," in Proc., IEEE Globecom Workshops, Houston, TX, Dec. 2011, pp. 389 - 393.

[17] A. Bartoli, J. Hernández-Serrano, M. Dohler, A. Kountouris, and D. Barthel, "Low-power low-rate goes long-range: The case for secure and cooperative machine-to-machine communications," in Proc, Workshop on Wireless Cooperative Network Security (WCNS), Valencia, Spain, May 2011

[18] C.-Y. Tu, C.-Y. Ho, and C.-Y. Huang, "Energy-efficient algorithms and evaluations for massive access management in cellular based machine to machine communications," in Proc., IEEE Veh. Technology Conf. (VTC), San Francisco, CA, Sep. 2011.

[19] A. S. Lioumpas and A. Alexiou, "Uplink scheduling for machine-tomachine communications in LTE-based cellular systems," in Proc., IEEE Globecom Workshops, Houston, TX, Dec. 2011, pp. 353 - 357.

[20] B. Prabhakar, E. U. Biyikoglu, and A. El Gamal, "Energy-efficient transmission over a wireless link via lazy packet scheduling," in Proc., IEEE INFOCOM, Anchorage, Alaska, Apr. 2001, pp. 386 - 394.

[21] A. El Gamal, C. Nair, B. Prabhakar, E. Uysal-Biyikoglu, and S. Zahedi, "Energy-efficient scheduling of packet transmissions over wireless networks," in Proc., IEEE INFOCOM, New York, Jun. 2002.

[22] I. Keslassy, M. Kodialam, and T. V. Lakshman, "Faster algorithms for minimum-energy scheduling of wireless data transmissions," in Proc., Modeling and Optimization in Mobile, Ad Hoc and Wireless Networks, INRIA Sophia-Antipolis, France, Mar. 2003.

[23] D. Slepian, "Bounds on communications," The Bell System Technical Journal, vol. 42, no. 3, pp. 681-707, May 1963.

[24] M. J. Neely, "Intelligent packet dropping for optimal energy-delay tradeoffs in wireless downlinks," IEEE Trans. on Automatic Control, vol. 54, no. 3, pp. 565 - 579, Mar. 2009. 